\title{
Comparative assessment of plasmid DNA delivery by encapsulation within or adsorbed on poly (D, L-lactide-co- glycolide) nanoparticles
}

\author{
Abd Almonem Doolaanea ${ }^{1,2 \star}$, Nur 'Izzati Mansor', Nurul Hafizah Mohd Nor', \\ Mohd Affendi Bin Mohd Shafri ${ }^{3}$, Farahidah Mohamed ${ }^{1,2,4}$ \\ ${ }^{1}$ Department of Pharmaceutical Technology, ${ }^{2}$ IKOP Sdn. Bhd., Pilot Plant Pharmaceutical Manufacturing, Faculty of Pharmacy, \\ ${ }^{3}$ Kulliyyah of Allied Health Sciences, International Islamic University Malaysia, 25200 Kuantan, ${ }^{4}$ International Institute of Halal \\ Research \& Training, Kulliyyah of Engineering, International Islamic University Malaysia, PO Box 10, 50728, Kuala Lumpur, \\ Malaysia
}

*For correspondence: Email: abdalmonemdoolaanea@yahoo.com; Tel: +60136238628; Fax: +609 5716775

\begin{abstract}
Purpose: To compare the gene delivery effectiveness of plasmid DNA ( $p D N A)$ encapsulated within poly (D,L-lactide-co-glycolide) (PLGA) nanoparticles with that adsorbed on PLGA nanoparticles. Methods: PLGA nanoparticles were prepared using solvent-evaporation method. To encapsulate PDNA within the particles, it was first complexed with cetyltrimethylammonium bromide (CTAB) and then added to the oil phase during the synthesis. For the adsorption, PLGA nanoparticles were first modified with either CTAB or chitosan and then PDNA was adsorbed on the particle surface by electrostatic interaction.

Results: Nanoparticles encapsulating pDNA exhibited better plasmid loading and protection with significantly lower burst release $(p<0.05)$ compared to that of the nanoparticles with adsorbed plasmid. Cell uptake of chitosan-modified nanoparticles by murine neuroblastoma (N2a) cells was significantly ( $p$ $<0.05)$ higher than that of chitosan-free nanoparticles. Nanoparticles encapsulating pDNA showed higher transfection efficiency $(p<0.05)$ in N2a cells.

Conclusion: Encapsulation of pDNA within PLGA nanoparticles presents a potential strategy for gene delivery that is superior to $P D N A$ adsorbed on the nanoparticle surface. In addition, encapsulation keeps the particle surface free for further modifications such as the addition of targeting ligands.
\end{abstract}

Keywords: Poly (D,L-lactide-co-glycolide), Plasmid DNA, Encapsulation, Adsorption, Cellular uptake, Gene therapy, Targeting ligands

This is an Open Access article that uses a funding model which does not charge readers or their institutions for access and distributed under the terms of the Creative Commons Attribution License (http://creativecommons.org/licenses/by/4.0) and the Budapest Open Access Initiative (http://www.budapestopenaccessinitiative.org/read), which permit unrestricted use, distribution, and reproduction in any medium, provided the original work is properly credited.

Tropical Journal of Pharmaceutical Research is indexed by Science Citation Index (SciSearch), Scopus, International Pharmaceutical Abstract, Chemical Abstracts, Embase, Index Copernicus, EBSCO, African Index Medicus, JournalSeek, Journal Citation Reports/Science Edition, Directory of Open Access Journals (DOAJ), African Journal Online, Bioline International, Open-J-Gate and Pharmacy Abstracts

\section{INTRODUCTION}

Gene therapy has shown promising results in the treatment of a wide range of diseases. Poly (D,Llactide-co-glycolide) (PLGA) is a biodegradable and biocompatible polymer [1]. Encapsulating plasmid DNA (pDNA) in PLGA microspheres protects it from enzymatic degradation and preserves its bioactivity and its ability to exhibit sustained release [2]. 
Microparticles and PLGA nanoparticles have been frequently used to deliver nucleic acids after being modified to bear positive charges. The positively-charged particles interact with the negatively-charged nucleic acids by means of electrostatic interactions. Some of the commonly used surface modifying materials are chitosan [3], cetyltrimethylammonium bromide (CTAB) [4] and poly-L-lysine (PLL) [5]. Another approach for delivering nucleic acids is by encapsulating them within PLGA microparticles or nanoparticles [6]. Every approach for encapsulation of PDNA may have its own advantages and disadvantages.

However, there are no available studies on the identification of comparative differences among the various methods of pDNA delivery. Furthermore, comparing different methods based on what is reported in the literature is arguably unreliable because different studies may use different materials obtained from different suppliers, which would vastly affect nanoparticle properties such as PLGA molecular weight and hydrophobicity, chitosan molecular weight and degree of de-acetylation, and fabrication procedure.

The present study was carried out to compare pDNA encapsulated in PLGA nanoparticles, with pDNA adsorbed onto PLGA nanoparticles, in terms of particle size, zeta potential, suspension stability, pDNA loading, release profile, cell uptake by neuron cells and the transfection efficiency.

\section{EXPERIMENTAL}

\section{Materials}

PLGA5004 [lactic to glycolic acid ratio of 50:50, intrinsic viscosity (IV) of $0.2 \mathrm{dl} / \mathrm{g}$, and acid terminated] was obtained from PURAC (Gorinchem, Netherland). Polyvinyl alcohol (PVA; MW 115 kDa, $88 \%$ hydrolysed) was purchased from BDH Laboratory Supplies (Poole, England). Dichloromethane (DCM), ethyl acetate (EA), cetyltrimethylammonium bromide (CTAB), low molecular weight chitosan (LCS) of $50-190 \mathrm{kDa}$ and medium molecular weight chitosan (MCS) of 190-310 kDa were obtained from Sigma-Aldrich (Steinheim, Germany). Glacial acetic acid (GAA) was product of Merck (Hohen-brunn, Germany). All other chemicals used in this study were of analytical grade unless otherwise stated. Plasmid DNA (pDNA)-encoding green fluorescent protein (GFP) was generously provided by Dr. Michael Bradbury (Mount Sinai School of Medicine, Department of Medicine, Manhattan, USA).

\section{Nanoparticle synthesis}

Five formulations (F1-5) of nanoparticles were synthesized by a modified emulsion solventdiffusion method [3]. The components of the formulations are presented in Table 1. Formulations F1-F4 were modified with either CTAB or chitosan in order to confer positive charge on the particles. Two of the formulations were modified with CTAB pre-dissolved in either aqueous phase (F1) or oil phase (F2). Another two formulations were modified by either LCS (F3) or MCS (F4). The oil phase was mixed with the aqueous phase (Table 1) and homogenised at about 20,500 rpm for 3 min using IKA® T10 basic homogeniser (IKA Werke $\mathrm{GmbH}$ and Co., Germany). The resulting emulsion was added to the dispersion medium and stirred for $2 \mathrm{~h}$ under vacuum. For F5, pDNA was encapsulated within the nanoparticles after complexing it with CTAB. The pDNA was complexed with CTAB at a 1:1 molar ratio, and the resultant precipitate was collected by centrifugation at $14,000 \mathrm{~g}$ for $5 \mathrm{~min}$, and rinsed with deionised water. The supernatant was discarded and the precipitated complex was dried at $50^{\circ} \mathrm{C}$ for $15 \mathrm{~min}$. The dried CTAB-pDNA complex was dissolved in glacial acetic acid (GAA) before being added to the oil phase. Like $\mathrm{F} 1$ and F2, the oil phase was homogenised with the aqueous phase and then added to the dispersion medium. The nanoparticles were collected by centrifugation, and washed and suspended in one millilitre of deionised water. Part of each formulation was kept in suspension form in ice bath whereas the other part was lyophilised. To calculate the concentration of the nanoparticle suspensions, aliquots of about 100 $\mu \mathrm{L}$ of the nanoparticle suspensions were centrifuged and the supernatants were discarded. This was followed by complete drying of the nanoparticles. Nanoparticle suspension concentration was calculated by dividing the weight of dried nanoparticles by the volume of the aliquot used $(100 \mu \mathrm{L})$.

\section{Nanoparticle characterization}

To determine the particle size and zeta potential, each nanoparticle suspension was diluted with deionised water and analysed by dynamic light scattering. For determination of the particle size, Malvern Zetasizer Nano Series Nano-S was used, while for the determination of zeta potential, Nano-Z was utilised (Malvern Instruments Ltd, Malvern, Worcestershire, UK). The polydispersity index (Pdl), which is a dimensionless number indicating the width of the size distribution (values range between 0 and 1), was also obtained from the particle size measurement. 
Table 1: Composition of the nanoparticle formulations

\begin{tabular}{|c|c|c|c|c|c|}
\hline Medium & $\mathbf{F 1}$ & F2 & F3 & F4 & F5 \\
\hline Oil phase & $\begin{array}{l}\text { PLGA: } 1 \% \text { w/v } \\
\text { DCM:EA (1:5) }\end{array}$ & $\begin{array}{l}\text { PLGA: } 1 \% \mathrm{w} / \mathrm{v} \\
\text { CTAB: } 1 \% \mathrm{w} / \mathrm{v} \\
\text { DCM:EA }(1: 1)\end{array}$ & $\begin{array}{l}\text { PLGA: } 1 \% \text { w/v } \\
\text { DCM:EA (1:5) }\end{array}$ & $\begin{array}{l}\text { PLGA: } 1 \% \text { w/v } \\
\text { DCM:EA (1:5) }\end{array}$ & $\begin{array}{l}\text { PLGA: } 1 \% \text { w/v } \\
\text { DCM:EA:GAA }(1: 4: 1) \\
\text { pDNA }^{a}: 1 \% \text { w/v }\end{array}$ \\
\hline $\begin{array}{l}\text { Aqueous } \\
\text { phase }\end{array}$ & $\begin{array}{l}1 \% \text { w/v PVA } \\
1 \% \text { w/v CTAB }\end{array}$ & $1 \%$ w/v PVA & $\begin{array}{l}1 \% \text { w/v PVA } \\
1 \% \text { w/v LCS }\end{array}$ & $\begin{array}{l}1 \% \text { w/v PVA } \\
1 \% \text { w/v MCS }\end{array}$ & $1 \% w / v$ PVA \\
\hline $\begin{array}{l}\text { Dispersion } \\
\text { medium }\end{array}$ & $1 \% \mathrm{w} / \mathrm{v}$ CTAB & $\begin{array}{l}\text { Deionised } \\
\text { water }\end{array}$ & $1 \% \mathrm{w} / \mathrm{v}$ LCS & $1 \% \mathrm{w} / \mathrm{v} \mathrm{MCS}$ & Deionised water \\
\hline
\end{tabular}

${ }^{a} p D N A$ already complexed with CTAB and dissolved in glacial acetic acid

For scanning electron microscopy (SEM), dried nanoparticles were sprinkled onto aluminium stubs pre-pasted with carbon adhesive tapes. Samples were sputter-coated with gold and viewed using Carl Zeiss Evoß 50 Scanning Electron Microscope (Oberkochen, Germany).

\section{Preparation and characterisation of nano- particle-pDNA (NP-pDNA) complexes}

Formulations F1-F4 were used to prepare NPpDNA complexes by adsorption of pDNA on the nanoparticle surface. An amount of $10 \mu \mathrm{g}$ pDNA from $1 \%(w / v)$ solution in Tris-EDTA (TE) buffer was mixed with nanoparticle suspensions. The total reaction volume was made up to $100 \mu \mathrm{L}$ with deionised water by gentle mixing using upand-down pipetting, and the mixture was incubated at room temperature for $1 \mathrm{~h}$ without shaking. The amounts of nanoparticles were varied and yielded NP:pDNA weight ratios of $5: 1$, 10:1, 20:1 and 50:1. The complex formation was verified by gel retardation. The NP-pDNA complexes were also subjected to zeta potential measurement.

NP-pDNA complexes and pDNA encapsulated F5 were examined for their ability to protect pDNA from serum by agarose gel electrophoresis as described by Agarwal et al [7]. Foetal bovine serum (FBS) was added to the NPpDNA complexes to a final concentration of $10 \%$ FBS and incubated at $37^{\circ} \mathrm{C}$ for $1 \mathrm{~h}$ or $24 \mathrm{~h}$. Immediately following the incubation, $5 \mu \mathrm{L}$ of 0.5 $M$ EDTA was added and the samples were placed on ice to inhibit DNA nuclease. The control was naked pDNA-treated as NP-pDNA complexes. The samples and the control were loaded on agarose gel and run for $1 \mathrm{~h}$, as described earlier [7].

\section{Release profile study}

NP-pDNA complexes at NP/pDNA ratio of 20:1, and $\mathrm{F} 5$ were subjected to release profile study in phosphate buffer $\left(\mathrm{pH} \mathrm{7.2)}\right.$ at $37^{\circ} \mathrm{C}$ with shaking at $120 \mathrm{rpm}$ to avoid nanoparticle precipitation, using a rotary incubator shaker (Innova 4000; New Brunswick Scientific Inc., Edison, NJ). The nanoparticles were collected by centrifugation at seven pre-determined time intervals $(3 \mathrm{~h}, 6 \mathrm{~h}, 12$ h, 1 day, 3 days, 7 days, and 14 days). Then, the supernatant fractions were used to quantify the released pDNA by measuring the absorbance at $260 \mathrm{~nm}$. Thereafter, the nanoparticles were re-suspended in equal volume of fresh buffer before being withdrawn and returned back to the incubator shaker.

\section{Cellular uptake of nanoparticles by murine neuroblastoma (N2a) cells}

Cellular uptake studies were carried out in accordance with the procedures earlier [8]. Nanoparticle formulations were prepared with inclusion of coumarin- 6 as a fluorescent probe. Murine neuroblastoma ( $\mathrm{N} 2 \mathrm{a})$ cells were seeded in a 96-well white flat-bottom microplate at a density of $2.5 \times 10^{5}$ cells per well and incubated for $48 \mathrm{~h}$ at $37^{\circ} \mathrm{C}$ and $5 \% \mathrm{CO}_{2}$. Nanoparticles were added to the cells at different concentrations: $5 \mu \mathrm{g}, 10 \mu \mathrm{g}$ and $20 \mu \mathrm{g}$ per 100 $\mu \mathrm{L}$. Following $1 \mathrm{~h}$ incubation, the cells were washed three times in ice-cold phosphate buffered saline (PBS) to remove the noninternalised nanoparticles. The cells were then visualised under an Olympus FSX100 fluorescence inverted microscope (Olympus, Tokyo, Japan). They were subsequently lysed with Promega cell culture lysis buffer (CCLR) (Promega; Madison, WI) and then lyophilised. This was followed by the solubilisation of coumarin-6 with methanol. Finally, the fluorescence intensity was measured in a fluorescence spectrophotometer (Perkin Elmer Corp., Norwalk, CT, USA) at $450 \mathrm{~nm}$ excitation and $505 \mathrm{~nm}$ emission.

\section{Transfection efficiency}

Transfection efficiency of pDNA adsorbed on the nanoparticles (F1-F4) or encapsulated within the particle (F5) was determined by monitoring the gene expression of GFP in N2a cells. Cells were seeded at a density of $25 \times 10^{5}$ cells per well in a 24-well flat-bottom microplate and incubated for $48 \mathrm{~h}$ at $37{ }^{\circ} \mathrm{C}$ and $5 \% \mathrm{CO}_{2}$. After that, the media were replaced with fresh media containing the 
nanoparticles and incubated for $48 \mathrm{~h}$. The cells were visualised in a Nikon Eclipse $\mathrm{Ti}$ fluorescence microscope and the captured images were analysed with Image $(\mathrm{NIH}$, Bethesda, MD, USA) to quantify the green colour produced by GFP.

\section{Statistical analysis}

Comparisons amongst the formulations were performed using analysis of variance (ANOVA) with Tukey's post hoc test. Values of $p<0.05$ were considered statistically significant. Minitab software version 16 (Minitab, State College, PA) was used to perform the statistical analysis.

\section{RESULTS}

\section{Characteristics of nanoparticles}

With water as suspending medium, the formulation with CTAB-pDNA complex (F5) exhibited smaller particle size than the chitosanmodified nanoparticles (F3 and F4) but was not significantly different from the formulation with CTAB in oil phase (F2, Figure 1). The suspending medium of $10 \%$ FBS strongly affected the particle size and the Pdl of chitosan- modified nanoparticles (but not chitosan-free nanoparticles, i.e., F1, F2 and F5). The particle size of MCS-modified nanoparticles (F4) was more affected by serum: it dropped down to resemble the non-modified nanoparticles (F5). One important feature of all formulations was that they did not exhibit any detectable aggregation in the presence of serum. This is an essential compatibility pre-requisite for in vivo administration.

Complexation of nanoparticles with pDNA resulted in significant changes in the particle sizes of F1, F3 and F4 but not F2. F1 complexed with pDNA underwent significant increase in both particle size and polydispersity. In contrast, F3 and F4 had significant reduction in particle size when complexed with pDNA. Interestingly, both formulations had similar particle size after complexation. Furthermore, F1, F3 and F4 revealed similar particle size when complexed with pDNA $(p>0.05)$.

The two formulations (F1 and F2) which contained CTAB as a cationic modifier, did not exhibit positive zeta potential (Figure 2). On the other hand, chitosan-modified nanoparticles (F3 and F4) were positively charged. F3 (with LCS)

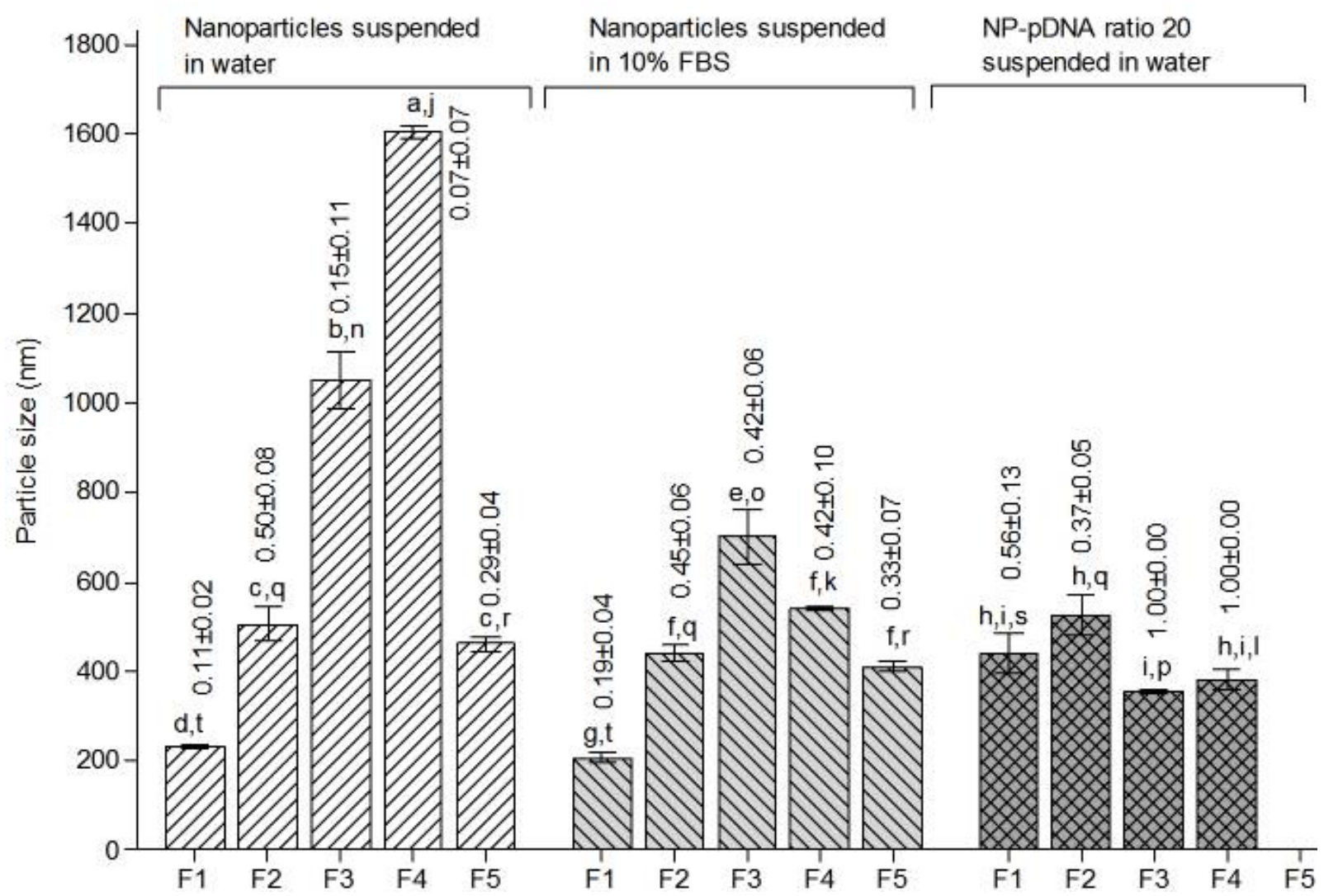

Figure 1: Particle size (mean \pm standard error) of the nanoparticles suspended in different conditions. Numbers over the bars are the Pdl \pm standard deviation (SD). Significant differences are represented by letters. Groups that do not share the same letter are significantly different $(p<0.05)$ 


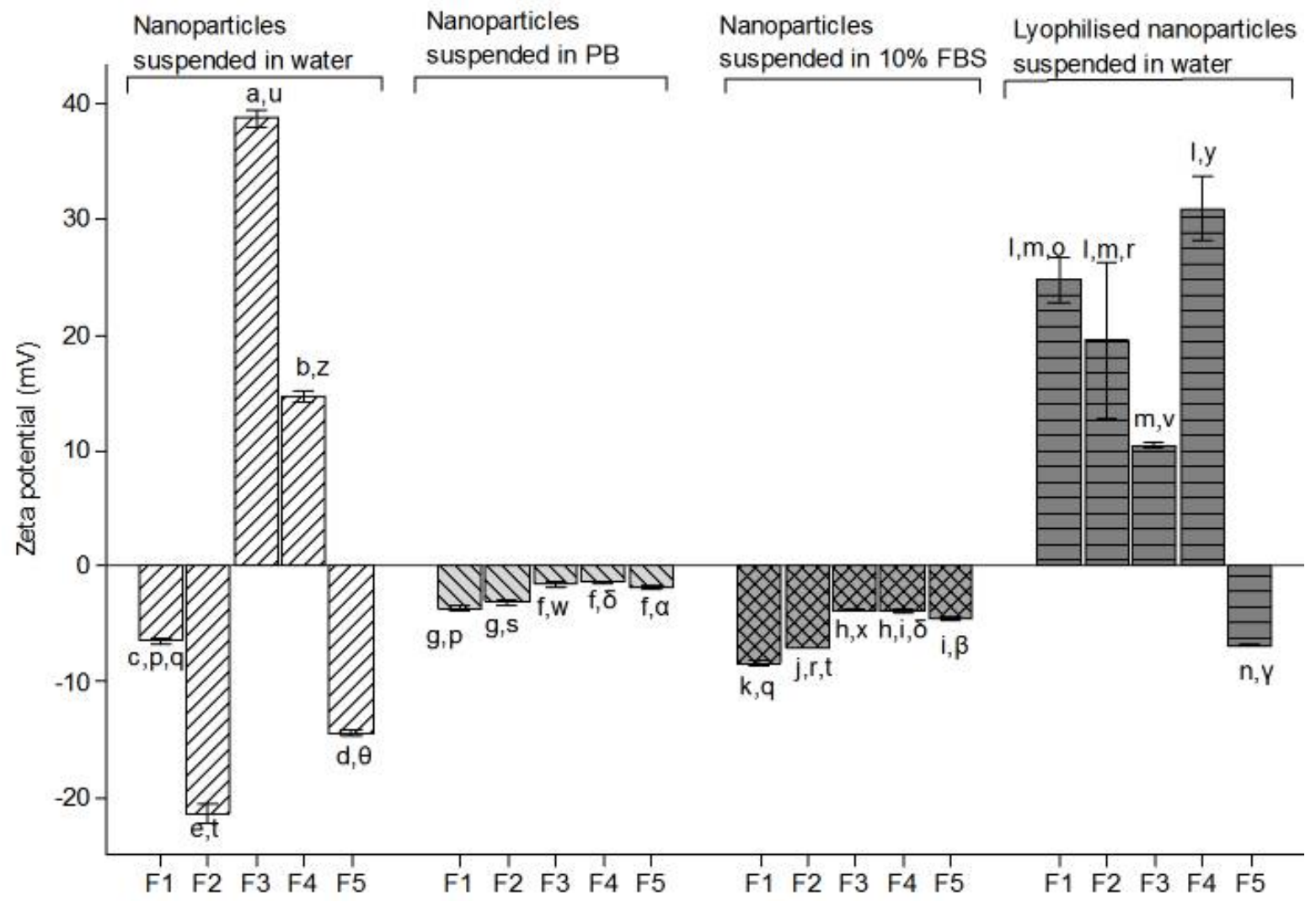

Figure 2: Zeta potential (mean \pm standard error) of the nanoparticle formulations after suspending in different conditions. Significant differences are represented by letters; groups that do not share a common letter are significantly different $(p>0.05)$

showed higher zeta potential. F5, in which CTAB was already complexed with pDNA, exhibited negative zeta potential. When the particles were suspended in phosphate buffer, $\mathrm{pH} 7.2$, the zeta potential of chitosan-modified nanoparticles ( $F 1$ and F2) was reversed to slightly negative. In the presence of $10 \%$ FBS, FBS was shown to confer a negative charge to all nanoparticle formulations with remarkably lower zeta potential in F1 and F2.

The zeta potential values before and after lyophilisation were compared. Interestingly, F1 and F2 manifested zeta potential inversion after lyophilisation with no significant difference (Figure 2). On the other hand, chitosan-modified nanoparticles remained positively charged after lyophilisation, but F3 zeta potential decreased while that of F4 increased. In contrast to CTABand chitosan-modified nanoparticles, F5 remained negatively charged after lyophilisation with slight decrease in zeta potential.

Scanning Electron Microscopy (SEM) revealed that the formulations had particles with smooth surfaces and without visible pores, as shown in Figure 3. The particle sizes of F3 and F4 under
SEM (Figure 3) appeared smaller than the size obtained by dynamic light scattering (Figure 1).
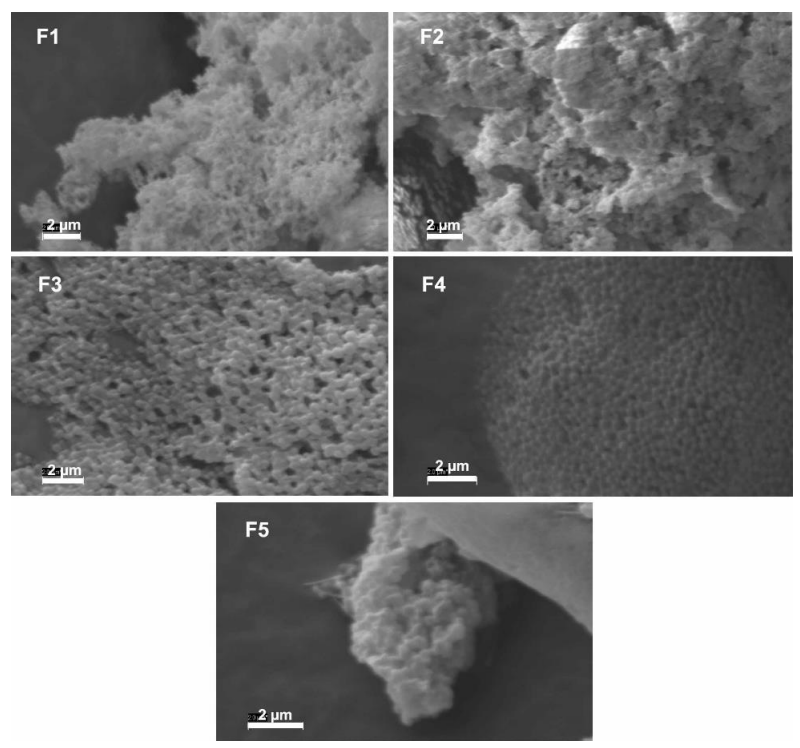

Figure 3: SEM images of nanoparticle formulations

\section{Characteristics of NP-pDNA complex}

CTAB- and chitosan-modified nanoparticle formulations F1-F4 were used to adsorb pDNA 
A

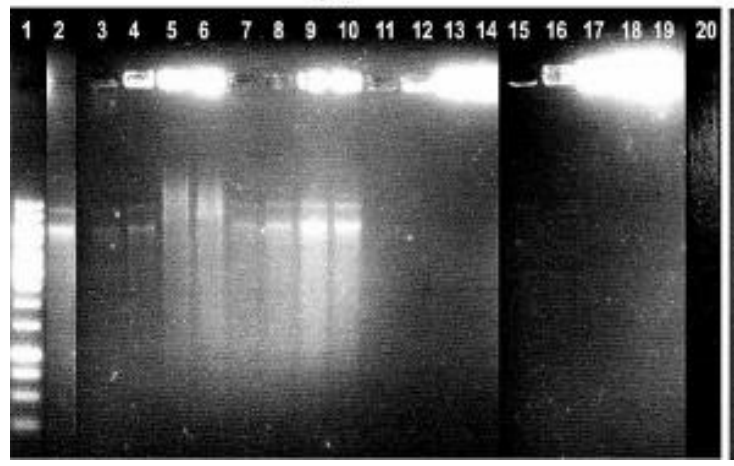

B
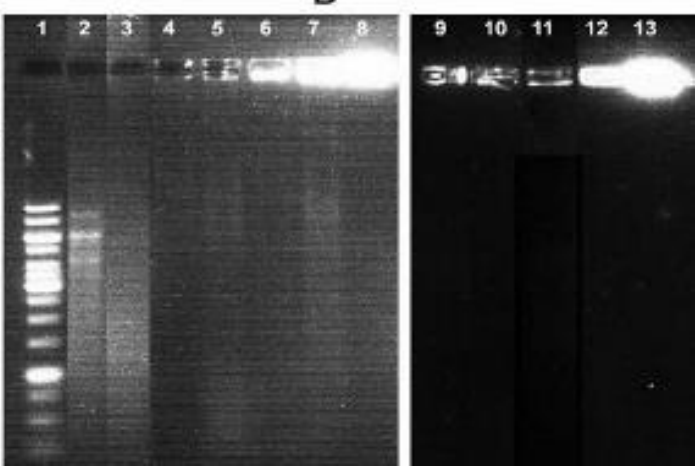

Figure 4: Characterisation of NP-pDNA complexes. (A) Gel retardation assay of the nanoparticles complexed with pDNA at different NP:pDNA ratios. Lanes: (1) $1 \mathrm{~kb}$ ladder, (2) naked pDNA, (3-6) F1-pDNA complexes at ratios 5:1, 10:1, 20:1, 50:1, respectively; (7-10) F2-pDNA complexes at ratios 5:1, 10:1, 20:1, 50:1, respectively; (11-14) F3-pDNA complexes at ratios 5:1, 10:1, 20:1, 50:1, respectively; (15-18) F4-pDNA complexes at ratios $5: 1,10: 1,20: 1,50: 1$, respectively; (19) F5, (20) CTAB-pDNA complex suspension. (B) Protection of pDNA in NPpDNA complexes at ratio 20:1 from degradation in $10 \%$ FBS. Lanes: (1) 1 kb ladder, (2) naked pDNA, (3) naked pDNA incubated in $10 \%$ FBS for $1 \mathrm{~h},(4-7)$ NP-pDNA ratio 20:1 complexes incubated in $10 \%$ FBS for $1 \mathrm{~h}$, (8) F5 incubated in $10 \%$ FBS for $1 \mathrm{~h},(9-12)$ NP-pDNA ratio $20: 1$ complexes incubated in $10 \%$ FBS for 24 h, (13) F5 incubated in $10 \%$ FBS for $24 \mathrm{~h}$

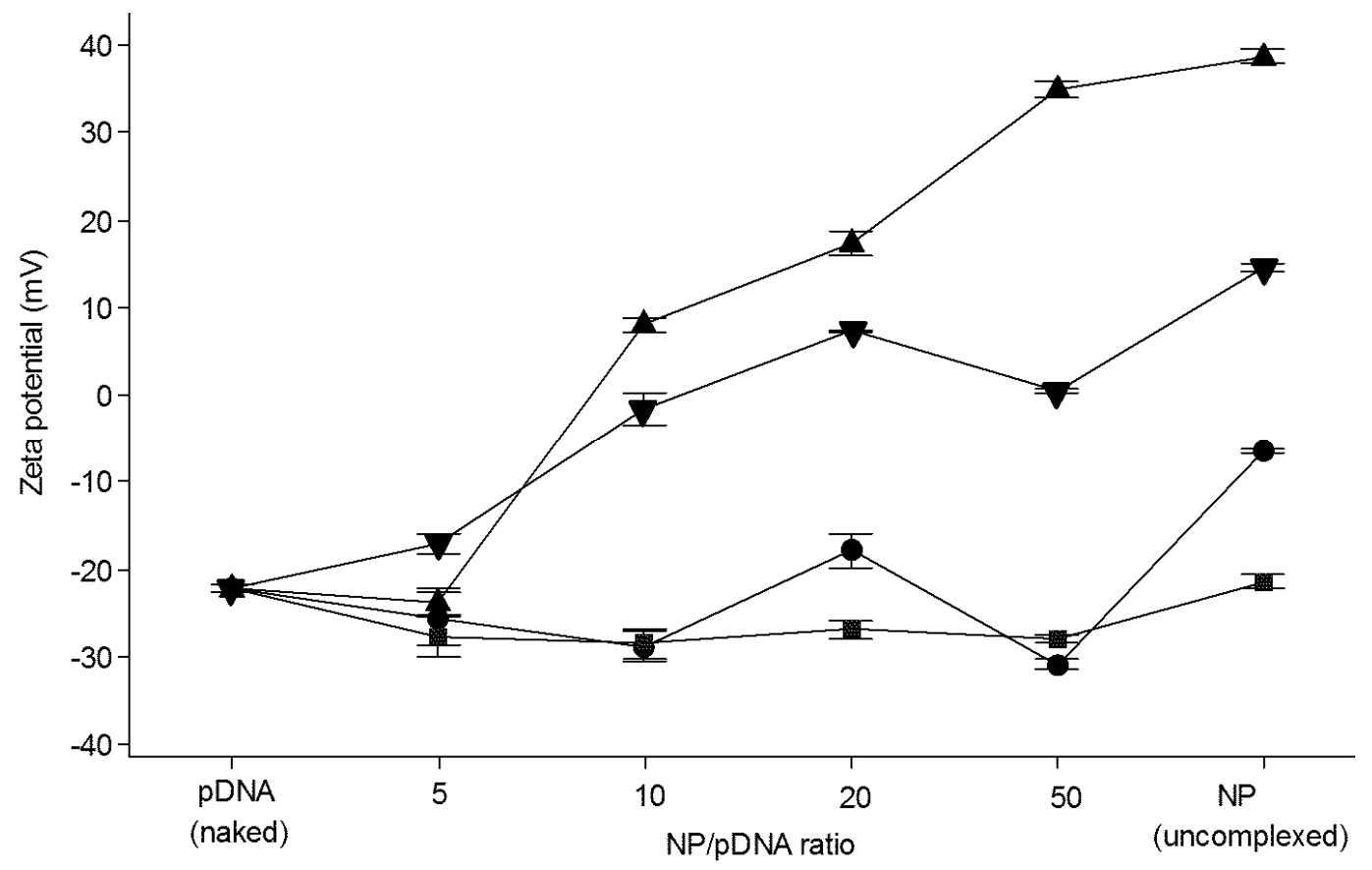

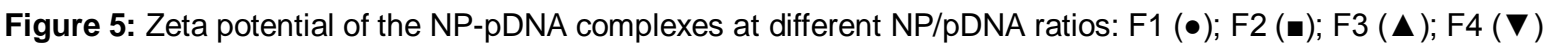

on the particle surface. The adsorption was evaluated by gel retardation (Figure 4) and zeta potential measurement (Figure 5) at different NP:pDNA ratios. Although $F 1$ revealed negative zeta potential, it could complex with pDNA but not completely, even at high NP:pDNA ratio. In addition, the pattern of pDNA migration on the gel at ratios 20:1 and 50:1 appeared similar to that when CTAB-pDNA complex was subjected to gel electrophoresis (lane 20 in Figure 4A). Zeta potential measurement revealed that $\mathrm{F} 1$ pDNA complexes at different ratios were negatively charged. F2, on the other hand, could not adsorb pDNA efficiently because free pDNA was detected in all ratios. Moreover, zeta potential showed negative charge that was similar in all ratios. In contrast, chitosan-modified nanoparticles (F3 and F4) adsorbed pDNA even at the lowest NP:pDNA ratio. In addition, zeta potential of the NP-pDNA complexes for F3 and F4 increased gradually with increase in NP:pDNA ratio.

The stabilities of the pDNA complexes were evaluated using agarose gel electrophoresis after incubating NP-pDNA complexes (ratio 20:1) in 
the presence of serum (10\% FBS) for $1 \mathrm{~h}$ and for $24 \mathrm{~h}$ (Figure 4B). The naked pDNA was completely degraded during the first hour of incubation. In contrast, F1-4 (lanes 4-7, Figure 4B) exhibited partial pDNA protection during $1 \mathrm{~h}$ incubation. F1 and F2 (lanes 4-5, Figure 4B) showed the least protection while F4 (lane 8, Figure 4B) showed the highest protection. On the other hand, F5 did not show any sign of smearing but only intense spot in the well. Overall, following $24 \mathrm{~h}$ incubation, only F4 and F5 conferred considerable stability on the pDNA.

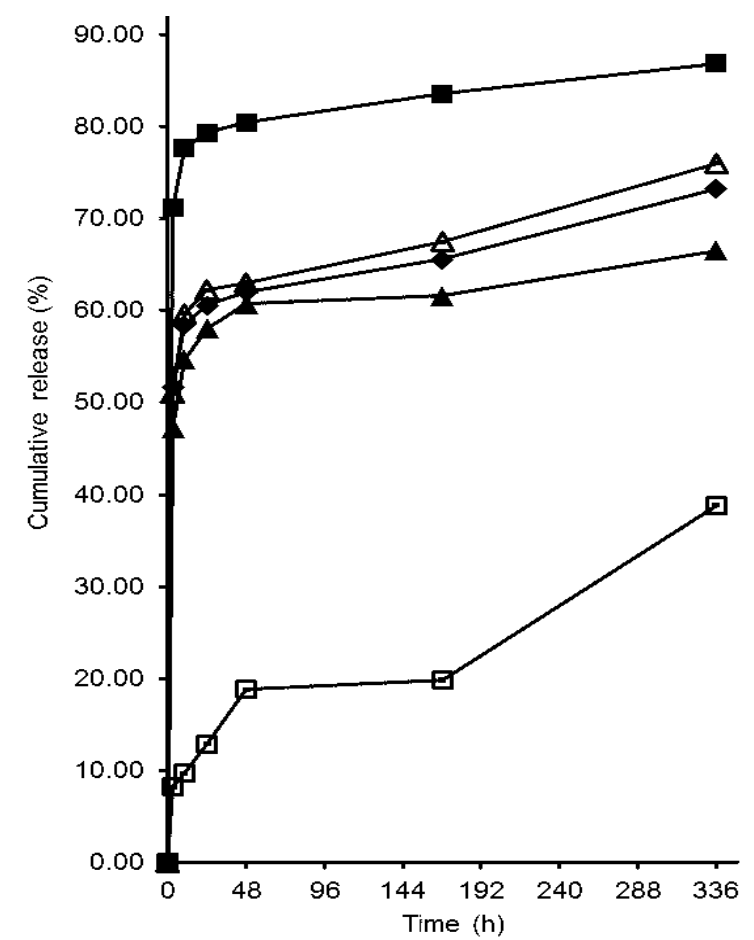

Figure 6: Release profile of F5 and NP-pDNA complexes at ratio 20:1 (nanoparticles: pDNA) over 2

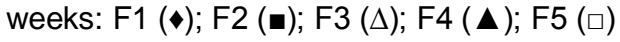

\section{Results from in vitro release study}

F5 and NP-pDNA complexes (ratio 20:1) were subjected to release profile study over 2 weeks (Figure 6). Plasmid DNA adsorbed on the particle surface (F1-F4) exhibited high burst release ( 50 - $80 \%$ within 24 h). F2, which poorly adsorbed and protected the pDNA, revealed the highest burst release $(79.4 \%$ within $24 \mathrm{~h})$ followed by slow release of the remaining pDNA. Interestingly, F2, F3 and F4 displayed almost similar burst release within $24 \mathrm{~h}(60.6,62.3$ and $58.1 \%$, respectively). On the other hand, F5 showed low burst release (only $12.9 \%$ within 24 h) followed by sustained release, with a total of $38.9 \%$ released over the 2 weeks.

\section{Cellular uptake of nanoparticles by N2a cells}

The results revealed that within each chitosanfree nanoparticle formulation (F1, F2 and F5), there was no significant difference in cellular uptake at all the tested concentrations (Figure 7 and Figure 8). In contrast, chitosan-modified nanoparticles (F3 and F4) showed increases in cellular uptake with increases in nanoparticle concentration.

\section{Degree of transfection of N2a cells}

Nanoparticles loaded with pDNA (encoding GFP), either by encapsulation (F5) or by adsorption (F1-F4), were used to transfect $\mathrm{N} 2 \mathrm{a}$ cells. F2 exhibited higher transfection than F1 (Table 2); the latter almost did not show any gene expression. Chitosan-modified

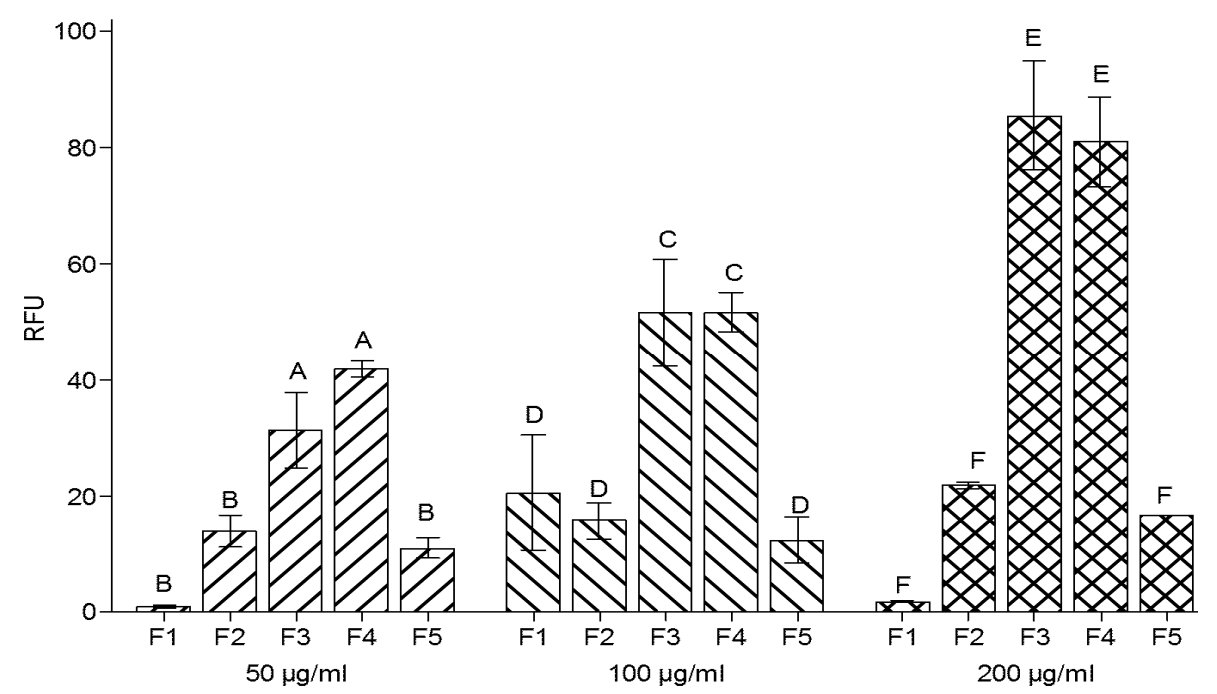

Figure 7: Quantitation of the cellular uptake of coumarin-6 loaded nanoparticles by N2a cells. Significance differences are represented by letters. Groups that do not share a common letter are significantly different $(p<0.05)$ 
nanoparticles exhibited different transfection efficiencies at different ratios; F3 and F4 showed the highest transfection at ratios 20:1 and 50:1, respectively. Amongst all formulations, F5 revealed the highest transfection. At the low NPpDNA ratio of $5: 1, F 2$ exhibited the highest transfection but at the high ratio of $50: 1$, F3 showed the highest transfection.

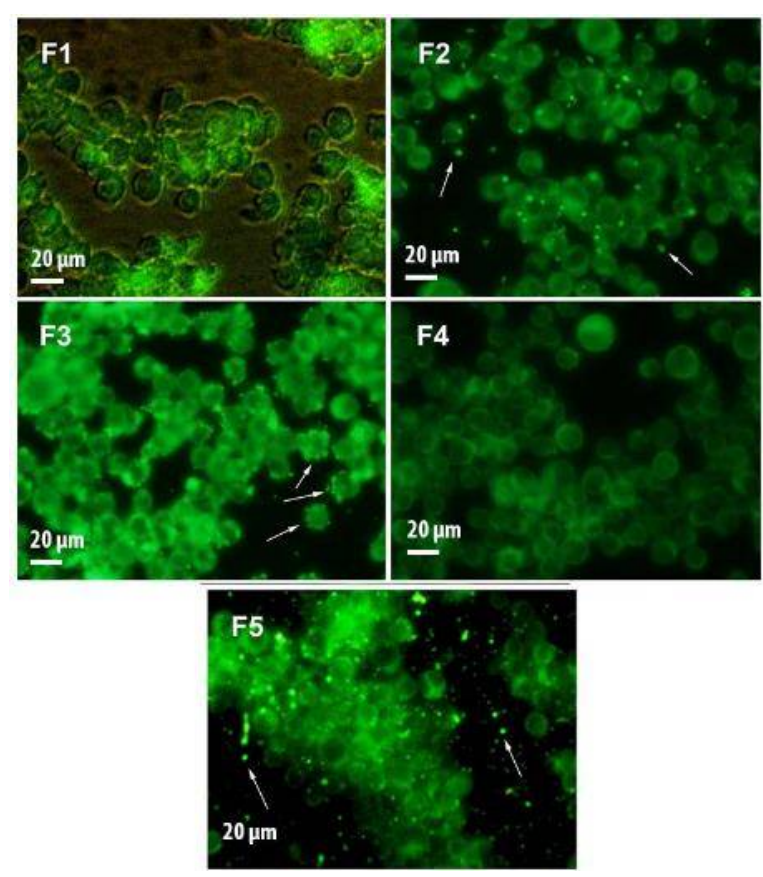

Figure 8: Cellular uptake of coumarin 6-loaded nanoparticles by $\mathrm{N} 2 \mathrm{a}$ as viewed by fluorescence microscopy. Arrows denote free particles present in the medium or adsorbed on the cell surface

\section{DISCUSSION}

Chitosan adsorption and the high viscosity of the aqueous phase were responsible for the increased particle size of chitosan-modified nanoparticle [9]. Serum contents could adsorb on the particle surface and may influence the particle size, polydispersity and zeta potential [11]. Schulze et al [12] reported deagglomeration while Nafee et al [13] reported agglomeration and increased particle size in the presence of serum. In contrast to the report of Nafee et al [13], it was found in this study that F3 and F4 exhibited decreased particle size in $10 \%$ FBS.

It has been suggested that chitosan adsorption on PLGA particles followed the multi-layer pattern with different conformations [9]. Serum components (zeta potential $=-16.7 \pm 0.7 \mathrm{mV}$ ) could adsorb on the nanoparticle surface causing chitosan to desorb, and its conformation could also become more compacted. Like the effect of serum, compaction of chitosan layers due to the adsorption of pDNA was responsible for the particle size decrease of chitosan-modified nanoparticles.

Table 2: Quantitation of transfection of N2a with pDNA (encoding GFP)-loaded nanoparticles

\begin{tabular}{|c|c|c|}
\hline Formulation & $\begin{array}{c}\text { NP:pDNA } \\
\text { ratio }\end{array}$ & $\begin{array}{c}\text { Transfection } \\
\text { efficiency }^{\mathrm{a}}\end{array}$ \\
\hline $\mathrm{F} 1$ & $\begin{array}{c}5: 1 \\
10: 1 \\
20: 1 \\
50: 1\end{array}$ & $\begin{array}{c}0 \pm 0(\mathrm{c}) \\
0.541 \pm 0.103(\mathrm{e}) \\
0.19 \pm 0.06(\mathrm{~h}) \\
0 \pm 0(\mathrm{j})\end{array}$ \\
\hline $\mathrm{F} 2$ & $\begin{array}{l}5: 1 \\
10: 1 \\
20: 1 \\
50: 1\end{array}$ & $\begin{array}{c}10.333 \pm 1.155(\mathrm{a}) \\
4.667 \pm 0.577(\mathrm{e}) \\
100.33 \pm 30.66(\mathrm{f}) \\
52.67 \pm 7.09(\mathrm{j})\end{array}$ \\
\hline F3 & $\begin{array}{c}5: 1 \\
10: 1 \\
20: 1 \\
50: 1\end{array}$ & $\begin{array}{c}0.593 \pm 0.090(\mathrm{c}) \\
28.0 \pm 4.359(\mathrm{~d}) \\
50.0 \pm 13.23(\mathrm{~g}) \\
142.33 \pm 46.32(\mathrm{i})\end{array}$ \\
\hline F4 & $\begin{array}{c}5: 1 \\
10: 1 \\
20: 1 \\
50: 1\end{array}$ & $\begin{array}{c}7.333 \pm 1.155(\mathrm{~b}) \\
25.667 \pm 4.041(\mathrm{~d}) \\
139.67 \pm 18.01(\mathrm{f}) \\
62.67 \pm 11.02(\mathrm{j})\end{array}$ \\
\hline $\begin{array}{l}\text { F5 } \\
\text { Untreated cells }\end{array}$ & - & $227.33 \pm 12.50$ \\
\hline $\begin{array}{l}\text { Untreated cells } \\
\text { Negative } \\
\text { control }\end{array}$ & $\begin{array}{l}- \\
-\end{array}$ & $\begin{array}{c}0.23 \pm 0.2 \\
0 \pm 0\end{array}$ \\
\hline $\begin{array}{l}\text { Positive } \\
\text { control }\end{array}$ & - & $0.86 \pm 0.14$ \\
\hline
\end{tabular}

Significant differences amongst different formulations of each of the NP:pDNA ratio were denoted by letters. Groups that do not share a letter differ significantly ( $p$ $<0.05$ ). F5 was significantly different from all other formulations with different ratios and also significantly different from positive and negative controls. ${ }^{a}$ Transfection efficiency was calculated as relative area of the green color in the captured images and reported as relative area $\times 1000$ )

Electrolytes cause compression of the double layers around the particles, leading to decrease in the absolute value of zeta potential [9]. This effect was clearly observed where all formulations had slightly negative zeta potential in phosphate buffer. In addition, the non-specific adsorption of serum components $[12,13]$ was responsible for the negative zeta potential of all formulations in $10 \%$ FBS. The inversion of zeta potential values of $\mathrm{F} 1$ and $\mathrm{F} 2$ could be explained by the re-localisation and re-orientation of CTAB. During the lyophilisation, CTAB molecules may migrate to the surface of the particles orienting their polar head to the surface in a manner similar to that of micelle conformation. The change of zeta potential of chitosan-modified nanoparticles after lyophilisation may be attributed to the change in chitosan conformation. In contrast, F5 exhibited the best stability of zeta potential before and after lyophilisation. 
A gene delivery vehicle should be able to protect pDNA under physiological conditions so that it can reach the target cell. The results of this study are in line with the general observation that high molecular weight chitosan produced more stable complexes with pDNA [14]. F5 offered the best protection to pDNA in the presence of serum due to encapsulation of the pDNA inside the particles. Furthermore, pre-complexation of pDNA with CTAB, followed by encapsulation within PLGA dramatically reduced the burst release of F5. This is of advantage because it would help to avoid the loss of pDNA before it reaches the target cell.

Particle surface plays important role in cellular uptake [8]. Chitosan-modified nanoparticles exhibited higher cell uptake despite the negative zeta potential in the presence of $10 \%$ FBS. Thus, there could be other factors involved in the enhancement effect of chitosan on cell uptake. Since nanoparticle uptake is the balance between endocytosis and exocytosis, chitosan may reduce the exocytosis aspect. The observation of free nanoparticles in the medium with F2 and F5 may be attributed to exocytosis since both formulations were chitosan-free.

The unexpected high transfection observed in F2 may be attributed to the weak interaction with pDNA. Similar results had been obtained by Köping-Höggård et al [15] who reported that polyplexes with easier dissociations yielded higher gene expression. The transfection efficiency of chitosan-modified nanoparticles is in line with the zeta potential measurements of the NP-pDNA complexes. The high stability of pDNA in F5 might be responsible for its high transfection efficiency. Thus, the transfection efficiency was a consequence of synergistic effects of different factors such as the strength of interaction between pDNA and nanoparticles, pDNA stability, zeta potential, cellular uptake and ease of pDNA dissociation inside the cell.

\section{CONCLUSION}

It is evident from the results obtained in the present study that the method of loading pDNA within PLGA nanoparticles influences the characteristics of the nanoparticles. This encapsulation loading method produces better results than the adsorption method. Encapsulation of pDNA as CTAB-pDNA complex in PLGA nanoparticles is a more effective technique for delivering pDNA for gene therapy than adsorption of pDNA on the particle surface. This encapsulation method also leaves the particle surface free for further modification that may even include addition of chitosan or targeting moieties.

\section{DECLARATIONS}

\section{Acknowledgement}

This work was funded by IIUM Research Initiative Grant Scheme (Grant no. RIGS15-0920092 and Grant no. RIGS16-325-0489) and Ministry of Science, Technology \& Innovation of Malaysia (MOSTI) (Grant no. 02-01-08-SF0101).

\section{Conflict of interest}

The authors declare that no conflict of interest is associated with this work.

\section{Contribution of authors}

The authors declare that this work was done by the authors named in this article and all liabilities pertaining to claims relating to the content of this article will be borne by them. A.A. Doolaaneaa, $\mathrm{NI}$ Mansor and NH Mohd Nor did the overall lab work. AA Doolaaneaa wrote the manuscript. MA Mohd Shafri supervised the cell culture. F Mohamed supervised the work and reviewed the manuscript.

\section{REFERENCES}

1. Lu JM, Wang X, Marin-Muller $C$, Wang $H$, Lin $P H, Y a o Q$, Chen $C$. Current advances in research and clinical applications of PLGA-based nanotechnology. Expert Rev Mol Diagn 2009; 9(4): 325-341.

2. Jang $\mathrm{JH}$, Shea $L D$. Intramuscular delivery of DNA releasing microspheres: microsphere properties and transgene expression. J Control Release 2006; 112(1): 120-128.

3. Doolaanea AA, Mansor NI, Mohd Nor NH, Mohamed F. Co-encapsulation of Nigella sativa oil and plasmid DNA for enhanced gene therapy of Alzheimer's disease. J Microencapsul 2016; 33(2): 114-126.

4. Basarkar A, Devineni $D$, Palaniappan $R$, Singh J. Preparation, characterization, cytotoxicity and transfection efficiency of poly(dl-lactide-co-glycolide) and poly(dl-lactic acid) cationic nanoparticles for controlled delivery of plasmid DNA. Int J Pharm 2007; 343(1-2): 247-254.

5. Blum JS, Saltzman WM. High loading efficiency and tunable release of plasmid DNA encapsulated in submicron particles fabricated from PLGA conjugated with poly-L-lysine. J Control Release 2008; 129(1): 6672.

6. Doolaanea AA, Ismail AFH, Mansor NI, Nor NHM, Mohamed F. Effect of Surfactants on Plasmid DNA Stability and Release from Poly (D, L-lactide-co- 
glycolide) Microspheres. Trop J Pharm Res 2015; 14(10): 1769-1778.

7. Agarwal A, Vilensky R, Stockdale A, Talmon Y, Unfer RC, Mallapragada SK. Colloidally stable novel copolymeric system for gene delivery in complete growth media. J Control Release 2007; 121(1-2): 28-37.

8. Doolaanea AA, Mansor NI, Mohd Nor NH, Mohamed F. Cellular uptake of Nigella sativa oil-PLGA microparticle by PC-12 cell line. J Microencapsul 2014; 31(6): 600608.

9. Guo C, Gemeinhart RA. Understanding the adsorption mechanism of chitosan onto poly(lactide-co-glycolide) particles. Eur J Pharm Biopharm 2008; 70(2): 597-604.

10. Lemarchand C, Gref $R$, Lesieur S, Hommel H, Vacher B, Besheer A, Maeder K, Couvreur P. Physico-chemical characterization of polysaccharide-coated nanoparticles. $J$ Control Release 2005; 108(1): 97-111.

11. Audouy S, Molema G, de Leij L, Hoekstra D. Serum as a modulator of lipoplex-mediated gene transfection: dependence of amphiphile, cell type and complex stability. J Gene Med 2000; 2(6): 465-476.

12. Schulze C, Schulze C, Kroll A, Schulze C, Kroll A, Lehr C-M, Schäfer UF, Becker K, Schnekenburger J, Schulze Isfort $C$, et al. Not ready to use - overcoming pitfalls when dispersing nanoparticles in physiological media. Nanotoxicology 2008; 2(2): 51-61.

13. Nafee $N$, Schneider $M$, Schaefer UF, Lehr C-M. Relevance of the colloidal stability of chitosan/PLGA nanoparticles on their cytotoxicity profile. Int $J$ Pharm 2009; 381(2): 130-139.

14. Mao S, Sun W, Kissel T. Chitosan-based formulations for delivery of DNA and siRNA. Adv Drug Deliv Rev 2010; 62(1): 12-27.

15. Köping-Höggård $M$, Vårum $K$, Issa $M$, Danielsen $S$, Christensen B, Stokke B, Artursson P. Improved chitosan-mediated gene delivery based on easily dissociated chitosan polyplexes of highly defined chitosan oligomers. Gene Ther 2004; 11(19): 14411452. 